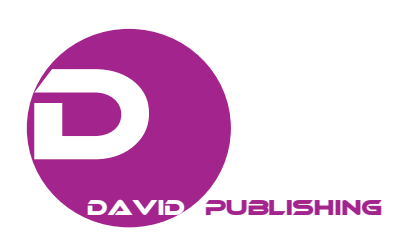

\title{
Usage of 3D Point Cloud Data in BIM (Building Information Modelling): Current Applications and Challenges
}

\author{
Tan $\mathrm{Qu}^{1}$ and Wei $\operatorname{Sun}^{2}$ \\ 1. School of Engineering, Design \& Construction, Seminole State College of Florida, Sanford SLM D103G, USA \\ 2. St. Johns River Water Management District, Palatka 32178, USA
}

\begin{abstract}
BIM (building information modelling) has gained wider acceptance in the A/E/C (architecture/engineering/construction) industry in the US and internationally. This paper presents current industry approaches of implementing 3D point cloud data in BIM and VDC (virtual design and construction) applications during various stages of a project life cycle and the challenges associated with processing the huge amount of $3 \mathrm{D}$ point cloud data. Conversion from discrete 3D point cloud raster data to geometric/vector BIM data remains to be a labor-intensive process. The needs for intelligent geometric feature detection/reconstruction algorithms for automated point cloud processing and issues related to data management are discussed. This paper also presents an innovative approach for integrating 3D point cloud data with BIM to efficiently augment built environment design, construction and management.
\end{abstract}

Key words: BIM, point cloud, laser scanning, 3D.

\section{Introduction}

$\mathrm{A} / \mathrm{E} / \mathrm{C} \quad$ (architecture/engineering/construction) industry has traditionally relied on $2 \mathrm{D}$ graphics to convey design and construction information. During the planning and design phases of a project, project design development generally evolves with progressive details in a 2D format except for occasionally generating conceptual 3D exterior models for architectural rendering purposes. Construction improvements, such as buildings, site and infrastructure elements, are abstracted into line or polyline representations in design documents. For example, on a structural framing plan, a W14×30 wide flange beam and a $\mathrm{C} 7 \times 12.2$ channel would be both represented graphically as single line elements with same plotting line weight while, in actuality, they differ in shapes and dimensions. When $3 \mathrm{D}$ drawings, such as

Corresponding author: Tan Qu, Ph.D., P.E., professor, research fields: construction automation, virtual design/construction and human computer interaction. E-mail: qut@seminolestate.edu. isometric diagrams, are developed, too often they are not drawn to dimension or scale and are not in the overall spatial context. Engineering analysis and computations are often based on the theoretical models and issues related to dimensional fit and space requirements are often unchecked. With the segmented $2 \mathrm{D}$ representations of the $3 \mathrm{D}$ build environment and building information being partitioned in various discipline drawings (i.e., building floor plans, roof plans, reflected ceiling plans, elevations, section views, foundation plans, structural framing plans, plumbing drawings, mechanical drawings, electrical drawings, etc.), detection of design errors usually is not an easy feat. Design errors and omissions uncaught until the field construction not only cause project delays, cost overruns or decreased productivities for the contractors but also constitute significant financial risks to the design firms when the project owners seek recovery of increased construction costs from the design firms. Similarly, for the participants involved in the construction process, namely construction field 
supervisors and workers, being able to interpret the information from the 2D construction documents and synthetize the segmented plan views, elevation views, cross section views and details mentally into a 3D representation of the project to detect constructability problems requires many years' training on the job and learned lessons.

The advent of BIM (building information modelling) and VDC (virtual design and construction) represented a quantum leap in the design and construction processes in the $\mathrm{A} / \mathrm{E} / \mathrm{C}$ industry. BIM and VDC differs fundamentally from the traditional 2D CAD (computer aided drafting) approach in terms of work flow and design technology, as they are multi-dimensional (spatial and temporal dimensions) model-based processes where the model not only contains the geometric information of the building elements but also metadata information related to physical and functional characteristics of the element, such as material specifications, cost and schedule information and other building lifecycle information. With BIM and VDC, design professionals in various disciplines can collaborate in the same model under the same spatial constraints without blind sight of other disciplines' work. Better multiparty communication and understanding from $3 \mathrm{D}$ visualization is the BIM benefit rated most likely to improve ROI (return of investment) [1].

Since the origin of the BIM concept, many applications have been found in the design and construction processes of $\mathrm{A} / \mathrm{E} / \mathrm{C}$ industry which include: design development phase inter-discipline spatial coordination; BIM elements quantity extraction for cost estimating; schedule-linked 4D BIM (time-dimension based BIM) for construction schedule simulation; constructability analyses; 5D BIM (4D $\mathrm{BIM}+$ Cost) for visualizing the progress of construction activities and the cost impact over time; as-built documentation; 6D BIM (as-built BIM + Operation and Maintenance Data) for facility management. McGraw-Hill Construction's 2012 BIM report [2] collected user ratings of BIM activities and processes. For design processes, users rated modelling building envelope by architects as the most frequently used design activity and as having a high value index with only moderate difficulty. For construction phases, spatial coordination tops value and frequency ratings for pre-construction activities and contractors found most BIM uses in constructability analysis and job planning. McGraw-Hill 2012 report did note, however, that interactive 4D and 5D BIM analysis remained to be an area struggled by most construction users. This may be conjectured in part to be due to that the visualization process in most 4D BIM applications does not necessarily reflect the exact process how the buildings are built on construction sites and many required temporary construction systems (formworks, shoring and support systems, vertical transportation systems, etc.) would add significant amount of modelling work and are very complex to be modelled in the contractors' BIM. This complexity involved with modelling the inclusion of temporary construction systems limits the widespread use of 4D BIM in constructability analyses due to cost implications. As a result, 4D BIM simulation with temporary construction systems (e.g., cranes and temporary support structures) is often only found on very large or complex projects where project budgets allow such uses.

The 3D geometric information contained in the BIM models generally reflects the "proposed" and "as-designed" condition even if it is developed during construction phase. In comparison, the $3 \mathrm{D}$ point clouds of the built environment provide a "reality" capture of the field conditions or as-built conditions. These 3D point cloud technologies naturally present themselves as much needed assistive solutions to augment the 3D VDC and BIM applications.

There has been a proliferation of academic research on extracting $3 \mathrm{D}$ as-built geometric information from point cloud technologies including mobile LiDAR, terrestrial laser scanning and photogrammetry in the $\mathrm{A} / \mathrm{E} / \mathrm{C}$ industry in the recent years. For example, 
Tang et al. [3] explored the usage of laser scanning in extracting bridge as-built data. Sepasgozar et al. [4] compared as-built information modelling using mobile and terrestrial LiDAR systems. However, there lacks of a synopsis of the current 3D point cloud data usage by the industry end users in various aspects of a construction project and the challenges associated with such usage.

\section{Methods and Materials}

This paper employed a combination of methods to obtain the data used for the study. This included literature review of the latest remote sensing and point cloud technologies industry reports, as well as interviews with industry end users of 3D point cloud data. Industry end users of 3D point cloud data in the $\mathrm{A} / \mathrm{E} / \mathrm{C}$ industry generally include design firms, construction companies, facility owners and 3D point cloud data service providers. The feedback from the 3D point cloud data service providers is particularly important as these users represent the actual technology users to produce the 3D point cloud data used by the downstream users in the supply chain relationship. The $3 \mathrm{D}$ point cloud data service providers are often the ones processing the raw point cloud data and extracting geometric information to re-construct BIM geometric models for as-build BIM creation using the state-of-the-art commercial 3D modelling solutions.

For this study, four industry 3D point cloud data users (Table 1) were interviewed on their current application of 3D point cloud data and the challenges they experienced in the application process. Each of the four users represented an important section of the
$\mathrm{A} / \mathrm{E} / \mathrm{C} /$ industry.

\section{Results and Discussions}

The results and discussions are organized in three areas: (1) review of current 3D point cloud data acquisition technologies; (2) usage of 3D point cloud data in the construction project life cycle; (3) challenges associated with implementing point cloud data in BIM.

\subsection{Available 3D Point Cloud Acquisition Technologies}

The term of 3D point cloud refers to cluster of discrete or raster points defined by $(x, y, z)$ coordinates in a $3 \mathrm{D}$ coordinate system that often represents the external surface of an object. Many technologies exist today to acquire 3D point clouds from a construction environment. They can be generally categorized as either range-based or image-based technologies.

\subsubsection{Range-Based Modelling}

Range-based 3D point cloud modelling or light detection and ranging (LiDAR) are hardware-based technology and generally use an active light source (laser or infrared light) and measures the time of flight of the signal or phase difference when the light signals are bounced back from objects. The 3D laser scanning, also known as TLS (terrestrial laser scanning), is a ground based LiDAR technology that measures the positions and dimensions of objects in the 3D space. In this technology, a laser beam is emitted from a laser light source on the scanner unit and used to scan the surfaces of the surrounding objects in a raster manner to create a 3D point cloud. There are generally two types of systems - impulse-based system

Table 1 List of 3D point cloud data users and user characteristics.

\begin{tabular}{llll}
\hline User types & 3D point cloud data application area & Annual business revenue \\
\hline User 1 & $\begin{array}{l}\text { ENR (Engineering News Record) } \\
\text { Top-20 international engineering } \\
\text { firm }\end{array}$ & Constructability analysis during design stage & $\$ 1,700,000,000$ \\
\hline User 2 & $\begin{array}{l}\text { Regional construction firm } \\
\text { management }\end{array}$ & Construability analysis during construction & $\$ 398,000,000$ \\
\hline User 3 & Fortune-500 company & Facility engineering analysis, virtual manufacturing & $\$ 15,600,000,000$ \\
\hline User 4 & $\begin{array}{l}\text { Regional 3D point cloud data } \\
\text { service provider }\end{array}$ & 3D point cloud data acquisition and modelling & $\$ 5,000,000$ \\
\hline
\end{tabular}


and phase-difference system. With impulse-based system, the distance between the scanner and the object is determined by multiplying half of the time a laser pulse travels to the object and back to the scanner with the speed of light (distance $=0.5 \cdot t \cdot v$, where, $t$ is time of travel and $v$ is the speed of light). The phase-difference based 3D laser scanners use a continuous laser beam instead of a laser pulse, to illuminate the surrounding environment to be scanned and determine the distance to objects by measuring the phase difference of the laser beam wave between the emitted state and received state. Accuracies of laser scanners at the present time are generally within $2 \mathrm{~mm}$ to $5 \mathrm{~mm}$. The 3D laser scanning has become a relatively matured technology and there are quite a few manufacturers producing this type of scanners. Examples include Faro, Leica, Riegel, Topcon, Trimble, Zoller and Frohlich and others. The 3D range cameras, also known as ToF (time-of-flight) cameras, are another subclass of LiDAR technology which allows 3D point clouds to be acquired at video frame rates. Compared to 3D laser scanners, 3D range cameras use infrared light as illumination source, instead of laser beam. Similar to 3D laser scanners, ToF cameras exist in two main variations: One measures distance by means of direct measurement of the travel time of an infrared light pulse using arrays of single-photon avalanche diodes; The other method uses amplitude modulated light and obtains distance information by measuring the phase difference between a reference signal and the reflected signal [5]. Examples of commercially available 3D range cameras include D-Imager, Fotonic, SwissRanger, XBOX Kinect, etc. Compared to laser scanners, 3D range cameras generally have shorter range (up to $60 \mathrm{~m}$ ) compared to laser scanners (up to $330 \mathrm{~m}$, e.g., Faro-X330) and lower resolutions. Rafibakhsh et al. [6] evaluated the use of XBOX Kinect sensor in construction environment and their results showed the point clouds generated from Kinect sensors have considerably lower resolution and depth accuracy than high-end terrestrial laser scanners.

\subsubsection{Image-Based Modelling}

As compared to ranged-based 3D point cloud technologies, image-based modelling is a mainly software-based technology and uses ordered or un-ordered digital photographs to generate 3D point clouds. Close-range photogrammetry is a photogrammetry technique used when the distance/range from the camera to the subject is short (generally within $300 \mathrm{~m}$ ) as compared to the aerial photogrammetry. The 3D coordinates of an object are calculated by the measurements made in two or more photographic images taken from different positions. With close-range photogrammetry, a calibrated camera and a precision control frame/network are essential to provide an accurate determination of the camera position, camera orientation, object point coordinates and calibration parameters. The photogrammetry modelling process is based on co-linearity equations and generally consists of: determination of the camera positions using bundle adjustment and control frame data; extracting of interest points on the object surfaces for the image matching process; image matching; determination of $(x, y, z)$ coordinates of surface point by bundle adjustment [7]. The accuracy of close-range photogrammetry method is generally within sub-millimeters. The SfM (Structure from Motion) is a computer vision-based image modelling method and is one of the proliferating research fields in recent years. SfM method uses a set of un-ordered and un-calibrated digital photographic images and processes them through a computer vision algorithms pipeline to reconstruct a 3D point cloud. The steps of SfM typically consists of automated feature detection and correspondence (matching) between the individual images to recover the extrinsic (rotation and translation) and intrinsic (focal length and distortion) camera parameters for estimating the 3D location of each identified feature and bundle adjustment to reconstruct dense point cloud [8].

Much academic discussions exist as to which type of 
3D point cloud technology is superior to others. The 3D laser scanning technologies require the time to set up the scanner unit and reference targets and each scan can generally take between less than 5 min and over 15 min depending on the level of quality and resolution setting selected. Multiple scan set-ups are almost always required and the number of set-ups is dependent on the size of environment to be scanned and, sometimes, it can take several days to obtain the point clouds for a complete coverage for large scale projects. In comparison, SfM-based image modelling methods can process ordinary photos that can be taken in a rapid and effortless fashion in a much short time frame, however, the successful reconstruction of the entire environment model and quality of point coverage from this method are not guaranteed. As such, laser scanning technology has been regarded as entailing high capital expenditure and being time-consuming to set up in the construction field and obtain scans [9, 10]. With the capability of acquiring up to one million points per second in real dimensions, laser scanners have the advantage to produce dense point clouds with high geometric accuracy in an automated manner by non-computer experts. In comparing laser scanning to SfM method, Skarlatos and Kiparissi [10] noted that, for small and medium size objects and distance, SfM seemed to have an advantage in terms of methodology and accuracy while, on large scale objects, laser scanning was better in terms of quality and processing time. In another similar comparative study, Golparvar-Fard et al. [9] found that, in both laboratory and actual field experiments, the accuracy of using image-based SfM modelling was less than the point cloud generated by laser scanner. As being a passive light user, image-based 3D point cloud technology requires adequate lighting condition in the scene. The proper image exposure and clarity or noise levels of pixels in the images are highly dependent on the lighting conditions in the scene and capabilities of the image sensor. Most of studies so far have been limited to daytime outdoor conditions or well-lid occupied building interiors where light conditions were not an issue and using pro-consumer level of digital cameras. However, performance issues of image-based 3D point cloud technologies in non-optimally lit construction interior space with temporary lighting as typically present during the construction stages were seldom evaluated. Range-based point cloud technologies are not affected by insufficient scene lighting conditions and can be operated in almost complete dark environments, although the camera unit integrated in the scanner will not be able to capture images of desired qualities and resolutions to render textural information for the acquired point clouds. In addition, the computer vision concepts and algorithms such as SIFT (scale-invariant feature transform) and MSV (multi-view stereo) being the foundation of the image-based 3D point cloud technology are very complex and not something easy to understand for most $\mathrm{A} / \mathrm{E} / \mathrm{C}$ industry users, therefore, can be intimidating. For these reasons, laser scanners despite the high initial capital costs are often selected for actual production uses by the industry point cloud data service providers.

\subsection{Usage of 3D Point Cloud Data in the Construction} Project Life Cycle

\subsubsection{Planning and Design Phases}

During the project planning and design stages, 3D point cloud technology can be used to quickly and inexpensively capture the surrounding environment in 3D format and the generated point cloud model can then be fused with proposed building BIM model to evaluate the construction impact to the existing neighborhood and check for potential siting issues. Foster et al. [11] demonstrated on a high-rise building project in a downtown area that $3 \mathrm{D}$ point cloud coupled with BIM allowed the design firm to conduct line of sight and shadowing studies and produce a 3D visualization of the construction impact to obtain the appropriate approvals to move forward with the project. On existing facility renovation/expansion projects, 3D point cloud technology can efficiently augment the 
virtual design and construction processes. On existing buildings with complex structures, laser scanning is often used to capture the intricate spatial geometry that would otherwise take a long time to model by traditionally manual measuring and modelling techniques.

\subsubsection{Bidding and Construction Phases}

Point cloud model of existing construction sites can be a virtual site/building survey tool to greatly assist contractors to better prepare construction estimates and bid proposals by minimizing the risks of missing scope items and misjudging the existing conditions. Traditionally, it has always been a challenge to sufficiently depict the existing conditions in a $2 \mathrm{D}$ survey where the existing features are often acquired manually and individually by using total station, GPS (global positioning system) or other types of surveying equipment. Cost of survey increases substantially when the level of survey details increases. Videorecording is a technique often used to document the conditions of the site prior to construction, but it does not lend the ability to retrieve any dimensional information from it. With point cloud technology, the contractor would be able to extract dimensional information and make realistic decisions as to the scope and complexity of the work required. Point cloud models captured at various stages of the construction integrated with the BIM also allow the contractors and design firms to conduct quick design compliance verifications and assess impacts and develop resolutions when deviations from design occur. The same point cloud models captured during the construction can be compared to the 4D BIM at the same time instance to assess the construction schedule performance as to whether the project is behind, on-target or ahead of the planned schedule. Much research efforts have taken place in this area. Bosche et al. [12], El-Omari and Moselhi [13], Turkan et al. [14] and Zhang and Arditi [15] investigated laser scanning point cloud technology whereas Golparvar-Fard et al. [8] presented SfM-based approach for implementation with 4D BIM for construction progress monitoring. There has also seen increased uses of 3D point cloud technologies with 4D BIM for constructability analyses. For example, Gilson and Mercure [16] documented CTDOT (Connecticut Department of Transportation) I-95 New Haven Harbor Crossing project where 3D point cloud model of existing conditions was integrated into the Navisworks ${ }^{\circledR} 4 \mathrm{D}$ BIM simulation to check for constructability issues between three levels of existing highway overpasses to be demolished and an historic building located just a few feet away (Fig. 1).

\subsubsection{Post-construction Phase}

One of the prevalent uses of 3D point cloud technologies at the present time is in the post-construction phase of a project. Project owners, recognizing the limitations of the existing 2D-based traditional as-built survey, are transitioning to point cloud based 3D as-built documentation. The as-built point cloud models are then either converted to BIM models on projects that did not start with BIM design or stored away for future use. On projects constructed from BIM, the as-built point cloud models are often used to update the construction BIM models. The acquired point clouds can be used for 4D constructability analysis of major equipment renewal/replacement during the FM (facility management) phase of the project life cycle where there is no longer adequate access for work area as compared to during the construction phase. Additionally, on buildings/structures that undergo movements due to service load or geotechnical conditions, time-lapse point cloud captures of the structure/building are used to produce before and after BIM models to determine the causes of movements and monitor the patterns and magnitude of the movement.

\subsection{Challenges Associated with Implementing Point Cloud Data in Building Information Modelling}

Despite of progresses made in implementing 3D point cloud technology in BIM, challenges remain in some areas of the integration process and are discussed below. 

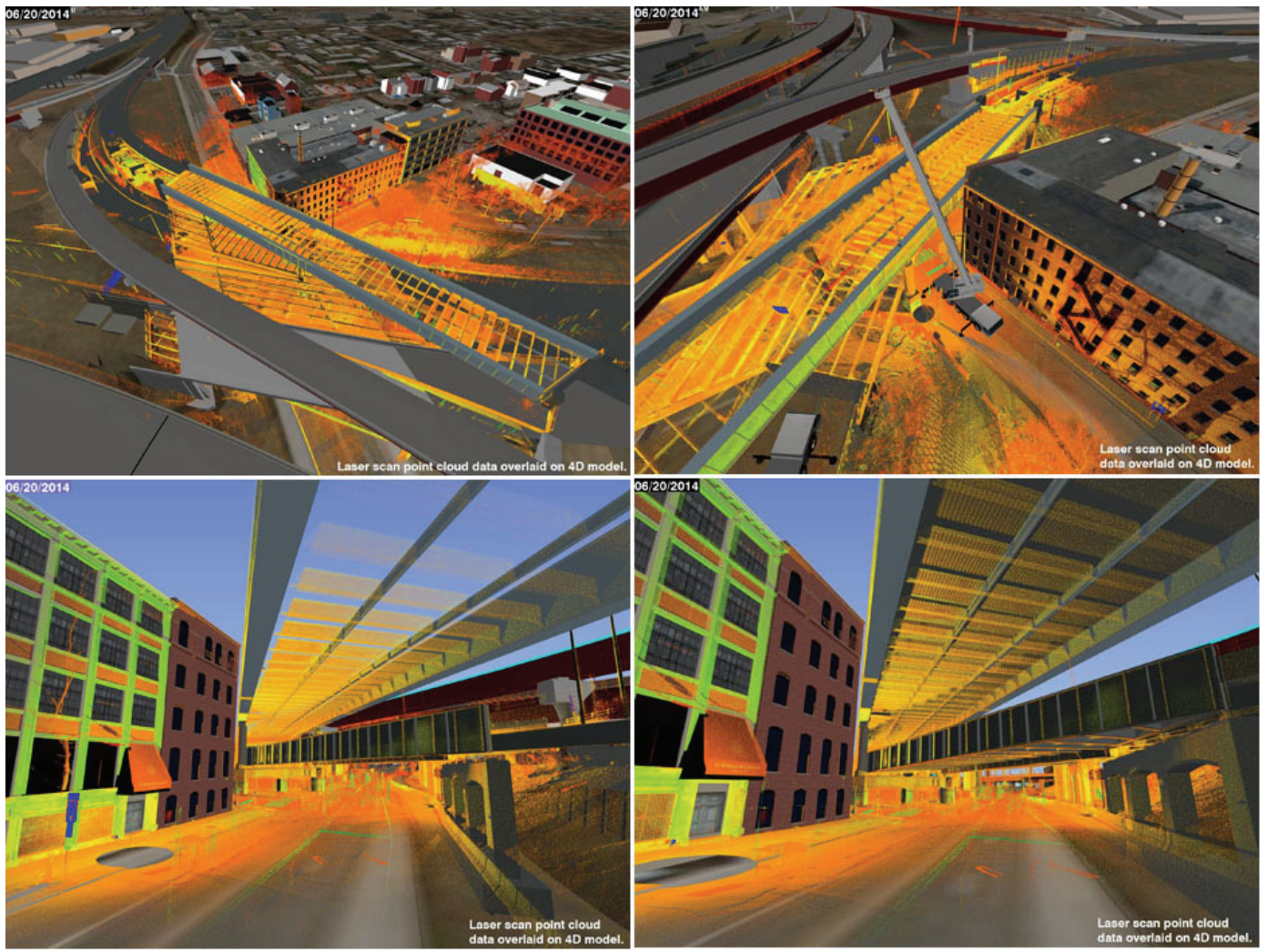

Fig. 1 Laser scan point cloud data overlaid on 4D model for constructability analysis on CTDOT I-95 New Haven Harbor Crossing project (images courtesy from CTDOT and Parsons Brinckerhoff).

3.3.1 Point Cloud Data vs. Building Information Modelling Data

Point cloud data generally consists of large amount of discrete point spatial coordinates $(x, y, z)$ plus the point intensity value for each point in the cloud, therefore, a point cloud model only contains raster geometric information. In comparison, a BIM model not only contains the geometric information but also includes a rich repertoire of building component properties, characteristics and management data. Due to raster nature of the point cloud model, it is not as efficient as the vector-based model used in BIM. Such $3 \mathrm{D}$ point cloud model data files are generally very large and require substantial computer storage and computational load. Table 2 shows a sample list of laser scanning projects and the characteristics of the point cloud files.

3.3.2 Point Cloud to Building Information Modelling Conversion

One of the driving forces behind the need for point cloud model conversion to BIM model is to produce printable 2D line drawings for construction field use. The raster nature of the point cloud is not suitable for producing construction plans and is not readily usable whereas in BIM the annotated typical floor plans, elevations, sections and details can be easily generated once the BIM model is completed. Because the technology adoption on construction sites generally is not at a level to allow for totally paperless construction sites and the majority of the construction field users are still accustomed to the traditional 2D paper-based construction documents, 2D paper-based plans will remain as the mainstay on construction sites for the foreseeable future.

One of the biggest challenges facing today is in the point cloud to BIM conversion process which often is very time-consuming. As a point cloud model only contains geometric information, its conversion means not only recreating the vector-based geometric model but also creating the BIM metadata from scratch which 
Table 2 List of sample projects with point cloud characteristics.

\begin{tabular}{|c|c|c|c|c|c|c|c|c|}
\hline Project name & Project type & Project size & $\begin{array}{l}\text { Number } \\
\text { of scans }\end{array}$ & $\begin{array}{l}\text { Raw file } \\
\text { size (GB) }\end{array}$ & $\begin{array}{l}\text { Number of } \\
\text { Points }\end{array}$ & $\begin{array}{l}\text { Working } \\
\text { pod export } \\
\text { size (GB) }\end{array}$ & $\begin{array}{l}\text { Field } \\
\text { man-hours }\end{array}$ & $\begin{array}{l}\text { Point cloud } \\
\text { registration \& } \\
\text { export man-hours }\end{array}$ \\
\hline $\begin{array}{l}\text { "X" pump } \\
\text { station }\end{array}$ & Civil & $\begin{array}{l}3 \text { stories/ } \\
4,500 \mathrm{SF}\end{array}$ & 38 & 5.19 & $618,339,207$ & $\begin{array}{l}6.65 \text { (at full } \\
\text { resolution) }\end{array}$ & 20 & 年 \\
\hline Mike's habitat & $\begin{array}{l}\text { Architectural/ } \\
\text { assets management }\end{array}$ & $\begin{array}{l}1 \text { story/ } \\
20,200 \mathrm{SF}\end{array}$ & 45 & 4.54 & $929,963,194$ & 15.4 & 20 & 14 \\
\hline $\begin{array}{l}\text { North } \\
\text { concessions } \\
\text { expansion }\end{array}$ & Architectural & $\begin{array}{l}5 \text { stories/ } \\
59,500 \mathrm{SF}\end{array}$ & 31 & 4.63 & $\begin{array}{l}112,987,322 \text { at } \\
1 / 2 \text { " spatial } \\
\text { filter }\end{array}$ & 1.8 & 10 & 8 \\
\hline Boiler & Industrial & 1 story & 10 & 1.31 & $436,978,820$ & - & 10 & 6 \\
\hline
\end{tabular}
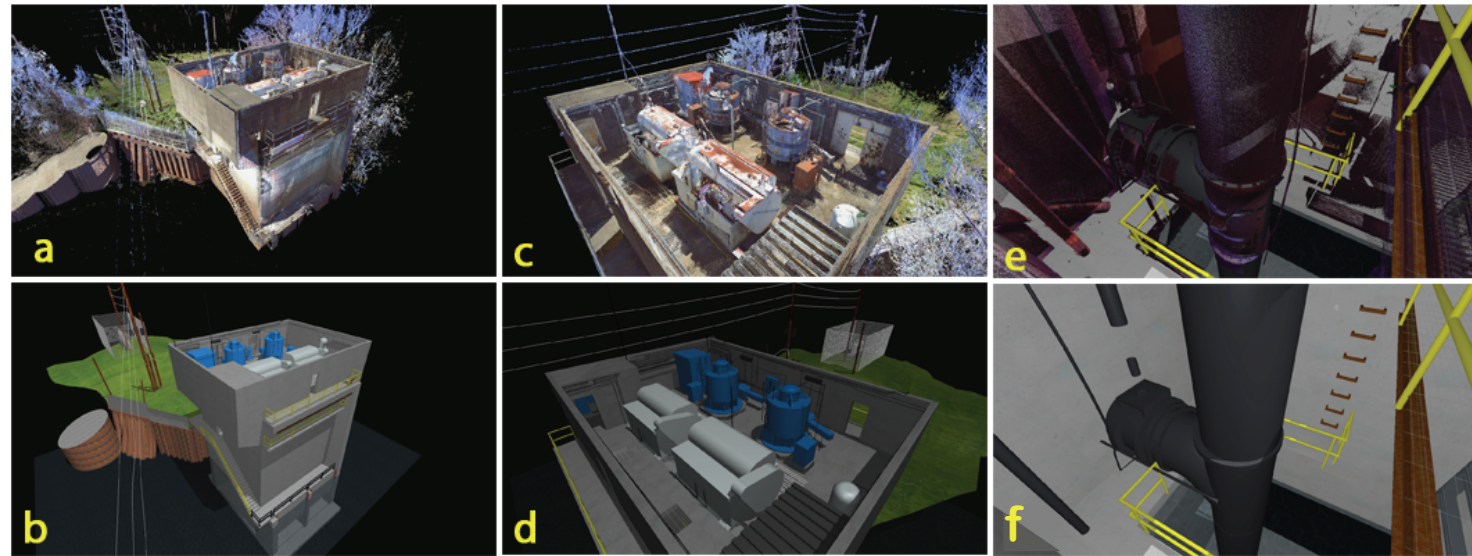

Fig. 2 Point cloud model of an existing pumping facility: (a) point cloud of the pump station; (b) converted BIM model; (c) motor center in point cloud; (d) motor center in BIM model; (e) intake shaft in point cloud; (f) intake shaft in BIM model.

can be a huge undertaking time-wise and cost-wise. Questions also arise on the idealization resulted in the conversion process. For example, a building exterior wall that is supposed to be flat according to the design may actually be slightly wavy as constructed due to tolerances achieved in the field. Should this wall be idealized as a flat surface during the point cloud to BIM conversion process or left as a non-flat surface as captured by the point cloud? At what deviation threshold that such idealization should be limited to? Point cloud data generally contains higher level of geometric details (attachment accessories, special features, fine architectural details and geometric deviations) than a typical BIM model even at LOD (level of development) 500 and modelling such details would add tremendous amount of man-hours. As such, some of the high-level details will inevitably have to be forfeited during the conversion process. With the all-inclusive nature of the reality capture, point cloud models often contain irrelevant or temporary features in the scene that would interfere with feature recognition operations during the modelling process. The conversion process is also affected by occlusions and partial point coverage issues (e.g., upper surfaces of overhead MEP system components under floor decks are generally not visible from the laser scanners or cameras therefore will have poor point coverage). Fig. 2 shows a point cloud model of an existing pumping facility that was obtained with laser scanning technology and converted to a BIM model. Some of high level of details, such as bolts on pipe flanges and plug valve actuation stem contained in the point cloud model, were omitted during the BIM object conversion process to reduce the modelling time. The lobe shaped motor drive components were manually modelled with composite shape geometry. A number of other features were omitted as well.

For these reasons, the conversion process is rarely 
fully automatic and often requires substantial time to manually extract geometric features. There are various commercial solutions (e.g., ClearEdge3D ${ }^{\circledR}, \mathrm{Kubit}^{\circledR}$, Geomagic $^{\circledR}$, Leica Cyclone ${ }^{\circledR}$, Bentley Decartes ${ }^{\circledR}$, $\mathrm{LFM}^{\circledR}$, etc.) available to automate some of the feature recognition and modelling processes, but they are currently limited to simple geometric shapes and objects (e.g., rectangular shaped windows and doors and straight circular pipes). Complex geometric shapes and objects commonly found in construction, such as open web bar joists and computational curve based building façade, would still require manual modelling efforts. With the continuous advancements in the computer vision research field, this challenge is anticipated to gradually ease with more or more automatic intelligent feature recognition and modelling tools becoming available.

\subsubsection{Data Management}

On some of the large projects or facilities where frequent $3 \mathrm{D}$ point cloud captures are required to track the project progress or facility changes, conversion from point cloud to BIM model can be very cost-prohibitive and the benefit-to-cost ratio may not be favorable for such endeavor. Data management of point clouds from different locations and scan dates can be a huge task. Registration precision of conjoining a large number of individual sets of point clouds and error propagation can greatly affect the quality of the overall project/facility point cloud model and detract the accuracy of the clash detection analyses.

\subsubsection{Hybrid Point Cloud + BIM Approach}

In certain applications, creating an as-built BIM model from hundreds of scanned point clouds was not only a huge cost endeavor but more importantly the lengthy time required to build such BIM model would render the model obsolete by the time it was finished. Instead, a hybrid point clouds + BIM model can be more robust and efficient. For example, for an automotive manufacturing plant owner, where the existing plant and associated equipment assemblies are represented by a progressive point cloud model, new equipment's 3D CAD model or new building addition BIM can be registered in the overall plant point cloud model. When an existing sub-assembly is to be relocated to other part of the manufacturing facility, point cloud for such sub-assembly would be segmented into a point cloud module and moved to destination location in the plant point cloud model. The updated plant point cloud model is then used with the $3 \mathrm{D}$ models of the new vehicles for clash detection and virtual manufacturing analyses. This approach was invented by Volvo Cars Group and found successful. In order to ensure the precise registration of the hundreds of incremental point clouds within the plant point cloud model, a factory coordinate system infrastructure was implemented which included a network of permanent laser target markers set at various locations of the plant. The coordinates $(x, y, z)$ of the permanent laser targets were verified and balanced with high precision surveying equipment. With the factory coordinate system infrastructure, precise alignment of a new point cloud cell with the plan point cloud model was possible and the projected average distance error was within $2.6 \mathrm{~mm}$.

\section{Conclusions}

This paper presents a review on the current implementation of 3D point cloud data in BIM and VDC by the industry end users. Hardware-based and computer vision algorithm based 3D point cloud modelling technologies are compared as to their advantages and disadvantages for use on construction sites. The 3D point cloud integration with BIM provides great benefits in facilitating the project planning and design process and offer the contractors the visualization tools in improving the project management performance. Challenges in $3 \mathrm{D}$ point cloud model to BIM model conversion process are discussed as the conversion process at the present time remains a labor intensive process where many improvements are needed in the area of automated feature detection tools in point cloud models. 
Challenges also exist in the area of data management and version control on projects that requires frequent 3D point cloud captures. Hybrid point cloud + BIM approach as discussed in Volvo case study offers an innovative approach on how point cloud technology can be useful in virtual design and construction in its native format.

\section{Acknowledgments}

The authors appreciate Connecticut Department of Transportation, Parsons Brinckerhoff and Forte and Tablada, Inc. for their support in providing project data and images.

\section{References}

[1] McGraw-Hill Construction. 2009. The Business Value of BIM: Getting Building Information Modelling to the Bottom Line. New York: McGraw-Hill Construction Smart Market Rep.

[2] McGraw-Hill Construction. 2012. The Business Value of BIM in North America: Multi-year Trend Analysis and User Ratings (2007-2012). New York: McGraw-Hill Construction Smart Market Rep.

[3] Tang, P., Huber, D., Akinci, B., Lipman, R., and Lytle, A. 2010. "Automatic Reconstruction of As-Built Building Information Models from Laser-Scanned Point Clouds: A Review of Related Techniques." Automation in Construction 19 (7): 829-43.

[4] Sepasgozar, S. M. E., Lim, S., and Shirowzhan, S. 2014. "Implementation of As-Built Building Information Modelling Using Mobile Lidar." Presented at 2014 Construction Research Congress, Georgia Institute of Technology, Atlanta, USA.

[5] Chiabrando, F., Piatti, D., and Rinaudo, F. 2010. "SR-4000 ToF Camera: Further Experimental Tests and First Applications to Metric Surveys." Presented at International Archives of Photogrammetry, Remote Sensing and Spatial Information Sciences, Part 5: Commission V Symposium, Newcastle Upon Tyne, UK.

[6] Rafibakhsh, N., Gong, J., Siddiqui, M., Gordon, C., and Lee, H. 2012. "Analysis of XBOX Kinect Sensor Data for
Use on Construction Sites: Depth Accuracy and Sensor Interference Assessment." Presented at 2012 Construction Research Congress, West Lafayette, USA.

[7] Ruther, H., Smit, J. L., and Kamamba, D. 2012. "A Comparison of Close-Range Photogrammetry to Terrestrial Laser Scanning for Heritage Documentation." South African Journal of Geomatics 1 (2): 149-62.

[8] Golparvar-Fard, M., Peña-Mora, F., and Savarese, S. 2009. "Application of D4AR - A 4D Augmented Reality Model for Automating Construction Progress Monitoring Data Collection, Processing and Communication." ITcon 14: 129-53.

[9] Golparvar-Fard, M., Bohn, J., Teizer, J., Savarese, S., and Peña-Mora, F. 2011. "Evaluation of Image-Based Modeling and Laser Scanning Accuracy for Emerging Automated Performance Monitoring Techniques." Automation in Construction 20 (8): 1143-55.

[10] Skarlatos, D., and Kiparissi, S. 2012. "Comparison of Laser Scanning, Photogrammetry and SfM-MVS Pipeline Applied in Structures and Artificial Surfaces." In ISPRS Annals of the Photogrammetry, Remote Sensing and Spatial Information Sciences, 299-304.

[11] Foster, B., Russo, J., Velazquez, C., and Pfeifle, S. 2013. Top 5 Ways Laser Scanning Can Save Money in the Design-Build Process. SPAR Industry Report.

[12] Bosche, F., Turkan, Y., Haas, C., and Haas, R. 2010. "Fusing 4D Modeling and Laser Scanning for Automated Construction Progress Control." Presented at 26th ARCOM Annual Conference and Annual General Meeting, Leeds, UK.

[13] El-Omari, S., and Moselhi, O. 2011. "Integrating Automated Data Acquisition Technologies for Progress Reporting of Construction Projects." Automation in Construction 20 (6): 699-705.

[14] Turkan, Y., Bosche, F., Haas, C. T., and Haas, R. 2012. "Automated Progress Tracking Using 4D Schedule and 3D Sensing Technologies." Automation in Construction 22: 414-21.

[15] Zhang, C., and Arditi, D. 2013. "Automated Progress Control Using Laser Scanning Technology." Automation in Construction 36: 108-16.

[16] Gilson, K., and Mercure, B. 2013. Virtual Design and Construction of Transportation Projects: 3D/4D Modeling Reduces Risk, Errors and Inefficiencies. Fayetteville: Civil Engineering News. 